\title{
Médiévales
}

Langues, Textes, Histoire

47 | automne 2004

Îles du Moyen Âge

\section{Un médiéviste historien des sciences}

Entretien avec Guy Beaujouan, Propos recueillis par Jean-Patrice Boudet, Joël Chandelier et Nicolas Weill-Parot

Jean-Patrice Boudet, Joël Chandelier et Nicolas Weill-Parot

\section{(2) OpenEdition}

12 Journals

Édition électronique

URL : https://journals.openedition.org/medievales/828

DOI : $10.4000 /$ medievales.828

ISSN : $1777-5892$

Éditeur

Presses universitaires de Vincennes

Édition imprimée

Date de publication : 1 décembre 2004

Pagination : 153-172

ISBN : 2-84292-157-7

ISSN : 0751-2708

\section{Référence électronique}

Jean-Patrice Boudet, Joël Chandelier et Nicolas Weill-Parot, « Un médiéviste historien des sciences », Médiévales [En ligne], 47 | automne 2004, mis en ligne le 02 avril 2007, consulté le 24 avril 2022. URL': http://journals.openedition.org/medievales/828 ; DOI : https://doi.org/10.4000/medievales.828

Ce document a été généré automatiquement le 24 avril 2022.

Tous droits réservés 


\section{Un médiéviste historien des sciences}

Entretien avec Guy Beaujouan, Propos recueillis par Jean-Patrice Boudet, Joël Chandelier et Nicolas Weill-Parot

Jean-Patrice Boudet, Joël Chandelier et Nicolas Weill-Parot

1 Pourquoi la science médiévale? Quel est votre parcours, ce qui explique que vous soyez spécialisé dans ce domaine?

2 Je me suis lancé dans la science médiévale à l'occasion d'une thèse d'École des chartes, que j'avais primitivement pensé faire sur l'histoire du vin d'Anjou, mais comme l'un de mes professeurs de première prétendait se lancer dans une thèse, que d'ailleurs il n'a pas faite, sur l'esprit de géométrie chez Pascal, il m'avait sensibilisé à l'histoire des sciences. De plus, j'ai eu moi aussi ma crise d'adolescence, mais au lieu de me droguer je faisais des mathématiques tout seul, ce qui ensuite m'a aidé à choisir une thèse sur l'histoire de l'arithmétique au Moyen Âge. J'ai choisi l'histoire de l'arithmétique parce que je songeais à faire une prospection systématique des manuscrits de la Bibliothèque nationale dans ce domaine déterminé. Alfred Cordoliani avait déjà fait la même chose pour le comput, pour lequel j'ai toujours eu une certaine répugnance, et l'arithmétique était donc pour moi le thème le plus facile à appréhender ${ }^{1}$.

3 Votre recherche portait au départ sur les mathématiques et plus précisément sur les manuscrits conservés à la Bibliothèque nationale, et cela rentrait dans le domaine de l'histoire des sciences. À cette époque, avez-vous eu des maîtres, ou bien vous êtes-vous formé tout seul ?

4 Je dois dire que lorsque j'ai commencé ce travail en 1944, le domaine de l'histoire des sciences, en particulier en France, était un véritable désert. J'ai souvent raconté comment j'étais allé voir Gaston Bachelard, qui était alors le grand patron de l'histoire des sciences. Il a eu l'extrême gentillesse de me recevoir chez lui toute une soirée, il a eu une conversation fascinante avec moi sur Berkeley et sur Malebranche, sur leur comparaison, mais ceci ne m'a servi à rien en ce qui concerne l'histoire des sciences médiévales, sinon à garder le souvenir que j'avais été un petit peu comme si Mozart avait joué du piano uniquement pour moi. Je raconte une autre anecdote, qui est la manière dont je suis entré en contact avec René Taton, qui est devenu après l'un des 
principaux organisateurs de l'histoire des sciences en France. Je cherchais désespérément l'unique exemplaire de l'histoire des mathématiques de David Eugene Smith qui se trouvait à la Sorbonne. Cet exemplaire n'était jamais accessible, et je me suis un jour rendu au service du prêt où l'on m'a dit : «Oui, c'est un certain M. Taton qui l'a emprunté. Il habite rue Gay-Lussac.» Je me suis dit: "Je vais aller le voir immédiatement! »C'est comme cela que j'ai débarqué chez Taton et que j'ai fait sa connaissance. Je vous raconte ces deux anecdotes pour vous montrer l'état désertique et désorganisé dans lequel était l'histoire des sciences en 1944. On se précipitait pour avoir le plus rapidement possible les revues américaines de l'époque de l'occupation qui ne se trouvaient dans aucune bibliothèque, pour avoir une certaine idée de ce qui se faisait récemment aux États-Unis.

Autrement dit, cela paraissait inédit d'être à la fois médiéviste et historien des sciences.

C'était une nouveauté. Je me souviens être allé visiter Alain de Bouärd, qui était professeur de paléographie à l'École des chartes, pour lequel j'avais beaucoup d'admiration et qui nous avait fait des éloges - que je trouve maintenant un peu excessifs - des travaux de Marcellin Berthelot sur l'histoire de l'alchimie. Je suis donc allé le voir, assez intimidé, en lui proposant mon sujet de thèse sur l'arithmétique. Il m'a répondu : «Sujet admirable, Monsieur, mais serez-vous capable de le faire?», ce qui évidemment ne relevait pas de la meilleure pédagogie pour encourager les élèves à faire des recherches!

Vous avez fait votre thèse des chartes et vous êtes ensuite entré à la Casa Velázquez.

8 L'un de mes grands protecteurs, en revanche, a été Charles Samaran et aussi Mario Roques. Après que j'ai d'abord travaillé pendant quelques mois pour gagner ma vie dans une banque, où je suscitais l'admiration de mes collègues en faisant très rapidement des opérations comme on les faisait au Moyen Âge, Samaran, avec sagesse, a trouvé que cette situation ne pouvait pas durer, et il m'a donc fait entrer aux Archives nationales à la section contemporaine, où j'évitais ainsi de me fatiguer les yeux et où il m'a aménagé des horaires qui me laissaient la possibilité d'aller travailler sur manuscrit à la Bibliothèque nationale. Cela a duré pendant trois ans, de 1947 à 1950, date à laquelle je suis entré à la Casa Velázquez avec l'idée de faire une prospection systématique des manuscrits scientifiques médiévaux occidentaux conservés dans les bibliothèques $\mathrm{d}$ 'Espagne, en particulier dans les bibliothèques ecclésiastiques.

Donc la Casa Velázquez, et ensuite?

10 Je suis de nouveau revenu aux Archives nationales, où j'étais à la section moderne dans les années 1952 à 1960 , et où j'avais pour spécialité d'aider les lecteurs qui venaient faire des recherches sur l'histoire des sciences à l'époque moderne, ce qui m'a donc donné des contacts qui m'ont beaucoup ouvert l'esprit, et là j'ai commencé à faire une espèce de guide ou de recensement des documents des Archives nationales concernant l'histoire des sciences. Ce rapport avec des gens travaillant sur la science à l'époque moderne a été très utile pour m'ouvrir l'esprit à l'égard de la science médiévale. J'ai passé les années 1960-1963 au CNRS, ce qui m'a permis de revenir largement en Espagne. Je suis donc entré aux Hautes Études en 1963, mais je faisais déjà des conférences auparavant, mon enseignement à l'École Pratique des Hautes Études comme chargé de conférences 10 heures par an ayant commencé en 1956. Comme directeur d'études, je suis resté à l'EPHE de 1963 à 1993, soit 30 ans. 
11 Avec le recul, est-ce que vous vous considérez vous-même avant tout comme un médiéviste ou comme un historien des sciences?

12 Je me considère comme les deux! Je suis peut-être considéré comme un mauvais médiéviste et comme un mauvais historien des sciences, du moins je me considère comme les deux.

13 L'un des problèmes auxquels vous avez été confronté réside dans les rapports parfois délicats, difficiles, entre l'histoire des sciences et l'histoire des mentalités, et dans une espèce de dialogue de sourds dont vous dites avoir souffert ${ }^{2}$. Dans un sens, on pourrait presque dire que vous avez vaincu, puisque l'histoire des mentalités n'est plus à la mode à l'heure actuelle, alors que l'histoire des sciences se porte plutôt bien. Mais que pensez-vous, avec le recul, de ce dialogue de sourds? Est-ce que finalement cela n'a pas débouché sur des choses positives?

Comme vous le dites, il y a eu une évolution dont je ne suis pas responsable mais que j'ai suivie avec plaisir. Lorsque j'ai commencé à dépasser un peu l'arithmétique, mon grand ennemi était Charles-Victor Langlois et le volume qu'il avait consacré à la connaissance de la nature et du monde dans la vie quotidienne au Moyen Âge ${ }^{3}$. Naturellement, lorsque je lançais cette affirmation, je choquais des médiévistes. Je me souviens de la réaction de Robert Fawtier qui était tout à fait surpris de me voir dire cela. Et effectivement, cette manière méprisante, pseudofolklorique, de considérer le regard des gens du Moyen Âge sur le monde me scandalisait beaucoup, et, dans toute la mesure du possible, j'ai réagi contre cela. Je vous donne un exemple typique que j'ai rencontré lorsque Claude Thomasset préparait sa thèse sur le dialogue de Placides et Timeo $^{4}$. Il s'agissait d'expliquer pourquoi, quand on s'élève en altitude, la température baisse alors que la sphère ultime est la sphère du feu. Lorsque l'on constatait que dans des textes comme Placides et Timeo, ce paradoxe était expliqué par un phénomène de ressort, on ricanait de la stupidité des gens du Moyen Âge, alors que ce fameux ressort n'est autre que l'antiperistasis d'Aristote, c'est-à-dire au contraire une explication extrêmement sophistiquée. J'avais une grande divergence de vue sur ce genre de sujets avec certains des avocats de l'histoire des mentalités qui reprochaient aux historiens des sciences (en particulier à Alexandre Koyré) de faire une histoire essentiellement conceptuelle, sans ouverture sociale et sans ouverture sur les "structures mentales", dont je n'ai jamais compris exactement ce que cela voulait dire.

Qu'est-ce que vous pensez de l'apport des philosophes des sciences, en particulier de l'apport de Pierre Duhem et d'Alexandre Koyrés?

Tout d'abord je dirai que Duhem n'est pas vraiment un philosophe des sciences, ni un historien. C'est un scientifique, un physicien, qui s'est intéressé à l'histoire des sciences, qui s'est passionné pour les manuscrits, qui a fait l'objet d'une véritable persécution du fait de son ultra-catholicisme et de la haine que lui portait Marcellin Berthelot, et qui, par conséquent, a travaillé en examinant des manuscrits de la Bibliothèque nationale par consultation à Bordeaux, c'est-à-dire en les regardant souvent avec précipitation. Je ne sais pas si vous pouvez publier cela, mais je n'ai pas un profond amour pour les historiens des sciences philosophes. Parce qu'il a été considéré pendant très longtemps que l'histoire des sciences était le domaine des philosophes, la commission du CNRS qui répartissait l'aide à l'histoire des sciences était une commission de philosophes, et, par conséquent, la porte n'était ouverte que parcimonieusement aux historiens des sciences de formation scientifique ou historienne. 
Oui justement, ce qui vous distingue très fortement de cette tendance, c'est votre contact de première main avec les sources, et même si, comme vous le dites, Pierre Duhem a eu des difficultés d'accès aux sources, il a quand même axé une grande partie de son Système du monde sur le contact direct avec les sources. La lecture de votre bibliographie dévoile un intérêt précoce et constant pour l'étude des manuscrits. Est-ce que vous pouvez nous expliquer les raisons de cette longue recherche? Est-ce une conséquence de votre formation de chartiste ou bien le plaisir de manipuler des sources inconnues était-il votre principale motivation?

Les deux. La formation a eu une grosse influence pour moi, d'autant plus que l'École des chartes de l'époque de la guerre avait peut-être un professorat moins ouvert qu'il ne l'est devenu - et encore, on pourrait en discuter - de telle sorte que j'avais une formation fondée sur le thème "pas d'histoire sans document » et sur le thème "ne rien écrire sans avoir lu la totalité de ce qui a été écrit sur le sujet ». Il est évident que ce sont deux maximes qui maintenant sont plus difficilement applicables, et moi-même j'avais la conviction que le progrès de l'histoire des sciences, c'était uniquement la découverte et l'exploitation de sources nouvelles. Et c'est lorsque j'ai lu le compte rendu de Koyré sur le livre d'Anneliese Maier, Die Vorlaüfer Galileis ${ }^{6}$, que je me suis rendu compte que l'histoire des sciences pouvait aussi progresser, même en ce qui concerne le Moyen Âge, par une analyse épistémologique plus fine de ce qu'était la révolution $\mathrm{du}$ xviie siècle; de sorte que cette espèce d'idolâtrie monopolistique du manuscrit s'est un peu effacée lorsque j'ai acquis une certaine maturité.

La question de l'origine de la révolution scientifique a aussi été l'une de vos préoccupations récurrentes. Vous avez souligné à plusieurs reprises l'arrière-fond idéologique de la divergence d'analyse entre Pierre Duhem et Koyré sur le rôle des condamnations de $1277^{7}$, tout en indiquant que les expériences de pensée secundum imaginationem doivent peut-être davantage à l'évolution de la logique depuis le milieu du XIII' siècle. Ce problème de la portée scientifique des condamnations de 1277 vous semble-t-il encore un champ d'étude pertinent? Comment voyezvous l'articulation entre la science scolastique et la science postérieure à la révolution galiléenne? Quelle place donner aux nouvelles voies de la physique au XIV siècle? Pensez-vous, au fond, que l'on puisse parler de révolution scientifique?

En ce qui concerne le problème des condamnations de 1277, on en a beaucoup parlé ces dernières années et le thème commence à s'user un peu. Au Moyen Âge, le domaine scientifique, la physique surtout, relevait, pour une large part, de la philosophie naturelle. Celle-ci cherchait souvent à fournir des explications profondes en se situant, si l'on peut dire, sur le plan de l'ontologie. La science des $x^{2} i^{e}{ }^{e}$ et $x v i i i^{e}$ siècles se contente d'explications causales plus modestes en essayant d'établir des relations fonctionnelles entre de simples phénomènes. La scolastique étudie les qualités comme des réalités en soi, alors que, à partir de la révolution galiléenne, la science moderne est fondée sur des mesures d'espace et de temps. Cela implique un recours tout différent aux mathématiques, même si les médiévaux admettaient généralement que, comme il est dit dans la Bible, Dieu a tout créé selon le nombre et la mesure. Si cette approche épistémologique peut sembler bien générale, l'exemple le plus frappant est le cas de la vitesse traitée au Moyen Âge comme qualité du mouvement. Je n'évoque pas le traditionnel contraste entre la méthode expérimentale de la science moderne et la prétendue limitation médiévale à l'expérience du sens commun: tant sur le plan épistémologique que dans la pratique, le problème est beaucoup plus complexe; je noterai seulement que l'optique d'Alhazen offre la plupart des caractéristiques données comme typiques de l'expérimentation moderne. 
21 À propos d'Oresme et de ces raisonnements secundum imaginationem, est-ce que vous voyez un lien avec la révolution scientifique?

Dans l'étude de ces raisonnements secundum imaginationem, il y a une évolution qui se produit actuellement et qui est un peu postérieure à ce que j'ai pu faire moi-même : c'est que, dans une certaine mesure, j'étais - mais Edward Grant l'était aussi ${ }^{8}$ - un héritier de Duhem en pensant que les condamnations de 1277 avaient libéré l'imagination au nom de la toute-puissance divine et encouragé les penseurs du xiv $^{\mathrm{e}}$ siècle à cette réflexion secundum imaginationem. Et c'est seulement à une date assez récente, en prêtant une attention beaucoup plus forte à la logique, qu'on s'est rendu compte que l'évolution de la logique médiévale était également porteuse d'un encouragement à ces raisonnements secundum imaginationem. Par exemple, en France je citerai l'un de mes élèves, Henri Hugonnard-Roche, qui, en partant de l'œuvre de Thémon Juif ${ }^{9}$ et en s'intéressant de plus en plus à la logique, a assez largement développé ce thème. Naturellement, il n'est pas le seul : Alain de Libera, Joël Biard et un certain nombre d'autres chercheurs, à une période relativement récente, ont mis l'accent sur cela. L'une des autocritiques que je me fais est de ne pas avoir eu le courage de me lancer suffisamment dans l'étude de la logique médiévale, parce que cela m'ennuyait : c'est un sujet assez aride.

Finalement, dans quelle mesure cette évolution de la logique au XIV siècle a-t-elle joué un rôle ou non dans cette révolution scientifique? Est-ce qu'on peut répondre à cette question sans être trop simpliste?

24 Les études du Père Wallace et d'Alistair Crombie ont très bien affiné les canaux de la relation entre cette logique du xiv ${ }^{\mathrm{e}}$ siècle et la révolution scientifique ${ }^{10}$. On a les cahiers de jeunesse de Galilée et on sait qu'un certain enseignement de la pensée médiévale dans le Collegium romanum est l'un des intermédiaires entre cette logique médiévale et la révolution scientifique. On cerne mieux l'approche par Galilée des problèmes posés au Moyen Âge. Maintenant on connaît tout de même assez bien de manière documentaire comment les choses se sont passées. Mais évidemment Galilée ne connaissait pas Oresme.

Abordons maintenant la question des rapports entre théorie et pratique, autrement dit celle de l'horizon de la science. À plusieurs reprises, ce problème des rapports entre le savoir théorique scientifique et la pratique technique vous a préoccupé11. L'origine de cette interrogation ne tientelle pas à la mauvaise réputation de la science médiévale, trop présente chez ceux que hante l'obsession de la modernité ? On a l'impression que l'on accuse la science médiévale d'être restée stérile en se référant davantage au regard des critères plus utilitaristes dérivés de la révolution scientifique qu'aux critères proprement médiévaux...

Je dois dire qu'historiquement ma sensibilisation à ce problème est passée par une autre voie. J'avais été invité par Michel Mollat, en même temps qu'Emmanuel Poulle, au premier colloque d'histoire maritime qui a eu lieu à Paris en $1956^{12}$. Nous trouvant, Emmanuel Poulle et moi-même, transportés dans l'historiographie portugaise des grandes découvertes, nous avions été frappés du fait que cette historiographie ne s'accordait pas du tout avec ce que nous connaissions de l'histoire des sciences au Moyen Âge, en particulier de l'histoire de l'astronomie. Et l'un des alibis portugais de ce décalage était l'évocation de la politique du secret. Autrement dit, sous prétexte que les connaissances scientifiques portugaises étaient couvertes par le secret, on pouvait dire un peu n'importe quoi. Par conséquent, il y avait un effort à faire pour reconsidérer ces choses. Cela a provoqué naturellement une très grande réaction portugaise ; j'ai eu des 
altercations, y compris avec le célèbre Armando Cortesaõ lors du colloque de 1960 sur Henri le Navigateur, mais peu à peu un dialogue s'est établi entre nous et des chercheurs plus raisonnables comme le commandant Teixeira da Mota, par exemple ${ }^{13}$. $C^{\prime}$ 'est donc à l'occasion de ce dialogue entre science et pratique dans les grandes découvertes maritimes que ma réflexion s'est de plus en plus orientée vers cela. Et l'existence même de l'alibi du secret dans cette problématique des grandes découvertes m'a naturellement orienté aussi vers les rapports entre la géométrie et l'architecture, à cause du prétendu secret des maîtres d'œuvre du Moyen Âge. Après, se posait aussi le problème du secret de l'alchimie et peu à peu ma réflexion s'est épaissie en m'interrogeant en particulier dans une conférence du Palais de la Découverte qui a eu un certain écho ${ }^{14}$, sur ce que disaient les gens du Moyen Âge, les traités encyclopédiques, Hugues de Saint-Victor, etc., des rapports entre théorie et pratique au Moyen Âge ; et j'ai beaucoup réfléchi à ces questions avec le regretté Bertrand Gille qui avait une remarquable connaissance de la technique médiévale ${ }^{15}$. J'ai essayé de réunir toutes mes réflexions en une espèce de testament, si j'ose dire, dans la contribution que j'ai donnée sur les rapports entre théorie et pratique dans le volume 4 de la grande histoire des sciences de l'Istituto della enciclopedia italiana, mais malheureusement je me trouve avoir parlé un peu dans le désert, du fait de la diffusion désastreuse de cette publication ${ }^{16}$.

Cela pose effectivement le problème des rapports entre théorie et pratique au Moyen Âge qui n'est absolument pas du même type que de nos jours.

Il y a d'abord le problème des historiens qui, lorsqu'ils s'occupent de technique, ne font pas l'effort de regarder quelles étaient les possibilités scientifiques établies, proposées aux techniciens, et qui entretiennent l'idée d'une sorte de rideau de fer entre les gens qui savent et les gens qui font. L'exemple le plus glorieux de mon existence à ce propos a été le calcul pour définir la hauteur du dôme de Milan qui a été fait en $1391^{17}$. Il s'agit d'un calcul d'expert pour évaluer ce que devait être la hauteur du dôme de Milan, mais ce calcul est conservé dans une copie extrêmement mauvaise et comportant des erreurs. Or Erwin Panofsky, qui est un immense historien de l'art médiéval, avait donné de ce calcul une interprétation absolument extravagante et j'avais montré qu'il fallait tout simplement corriger le texte qui nous est parvenu par une tradition manuscrite très mauvaise, et que le calcul aberrant que proposait Panofsky sous prétexte d'un certain romantisme du maître d'œuvre était en réalité un calcul tout à fait normal pour déterminer la hauteur d'un triangle équilatéral. Et là, l'une de mes grandes satisfactions a été de recevoir une lettre très aimable de Panofsky lui-même, reconnaissant qu'il s'était trompé.

Vous avez abordé également la question de l'histoire de la médecine en Espagne dans votre étude sur l'école médicale de Guadalupe ${ }^{18}$. Cette étude présente un versant pratique important, et il y avait à Guadalupe un hôpital. Dès lors, comment considérez-vous que la médecine, en tant que science mêlant théorie et pratique, s'intègre à votre réflexion plus vaste sur les rapports entre science théorique et application pratique au Moyen Âge?

Le cas de Guadalupe est un peu particulier dans la mesure où le problème se posait dans un milieu extra-universitaire, à l'intérieur d'un couvent resté en partie héritier de ce qu'était la médecine du haut Moyen Âge. Guadalupe se trouve en Extremadure, une région excentrée par rapport aux grandes universités. On pourrait peut-être m'accuser d'avoir, sinon une certaine aversion pour l'université, du moins un rapport un peu conflictuel avec elle, mais je considère que l'université n'a pas le monopole du génie 
scientifique, et qu'au contraire les préjugés et les cloisonnements universitaires sont souvent un frein au développement scientifique ou au développement de l'imagination scientifique ; et le cas de Guadalupe illustre cela dans la mesure où le fait de ne pas être prisonnier du galénisme, de la scolastique médicale, etc., a donné à ces gens une espèce de liberté qui avait des éléments négatifs mais aussi des éléments positifs.

Cela nous conduit au problème de l'innovation. Vous situez, dans un article qui a fait date, «la prise de conscience de l'aptitude à innover » dans les années 1260-1270, avec un certain nombre d'hommes de science majeurs, Albert le Grand, Campanus de Novare, Teodorico Borgognoni, Pierre de Maricourt, Roger Bacon, qui ont contribué à promouvoir d'une manière décisive mais ambiguë une certaine idée de progrès ou, en tout cas, d'évolution ${ }^{19}$. Pourquoi, si on peut le savoir, ces innovations ont-elles pris place globalement en dehors du cadre strict de l'université?

Oui, enfin, c'est un peu caricatural, parce que l'une de ces grandes innovations se rattache à l'école de Jordanus Nemorarius : ses innovations sur la statique ont, par exemple, donné les règles du levier coudé et du plan incliné, qui ne se trouvaient pas dans la science grecque, mais en réalité on ne sait à peu près rien de Jordanus et, par conséquent, le problème de son intégration ou de sa non-intégration à la science universitaire n'est pas du tout évident.

Dire « en dehors " serait aller trop loin, mais on a l'impression que vous insistez sur le fait que ce n'était pas forcément au cœur de l'université que ce processus a vu le jour, que c'était peut-être légèrement en marge.

Oui, mais les choses sont délicates. Je prends le cas d'Albert le Grand, qui malgré tout appartient à ce moment d'innovation par ses recherches sur la botanique, sur la zoologie etc., recherches qui sont donc un progrès et manifestent une certaine indépendance par rapport à Aristote. Ce qui est caractéristique, c'est qu'on aurait attendu que l'œuvre scientifique d'Albert le Grand soit antérieure à son œuvre théologique pour suivre le cursus normal, or en réalité son œuvre théologique est essentiellement parisienne, et c'est quand il va à Cologne, où il est donc plus indépendant de la pression du conditionnement d'une grande université comme celle de Paris, que sa créativité scientifique est la plus grande. Quant au De magnete de Pierre de Maricourt, c'est un cas évident de création extra-universitaire.

Pourtant la rationalité scolastique était un outil intellectuel particulièrement rigoureux et riche; or on a l'impression que ces innovations du milieu $d u$ XIII siècle s'effectuent, peut-être pas en dehors de ces sillons de la rationalité scolastique, mais un peu à côté.

Cette rationalité scolastique manifeste sa créativité scientifique plutôt au xive siècle qu'au xiiie siècle; au xiiie siècle, elle fait encore la part belle à des personnalités exceptionnelles.

37 Le lien que vous établissez, qui est quand même très intéressant, entre cette prise de conscience de l'aptitude à innover, la date de 1260 et les prophéties attribuées à Joachim de Flore, est quelque chose qui mériterait d'être creusé.

Il faut dire que le problème n'est pas un problème uniquement scientifique, parce que dans l'article auquel vous vous référe ${ }^{20}, j^{\prime}$ avais cité - d'ailleurs, je ne l'avais pas trouvé moi-même, c'était le Père Chenu qui me l'avait indiqué - des textes sur le caractère considéré comme très innovant de Thomas d'Aquin : le thomisme est considéré aussi à l'époque comme une innovation.

Venons-en à la question de l'enseignement des disciplines scientifiques à l'universitée ${ }^{21}$. À Paris, alors que les statuts de la Faculté des arts de 1255 montrent une Faculté orientée essentiellement 
vers la philosophie aristotélicienne (philosophie naturelle, métaphysique, éthique), rien n'est dit de l'enseignement des matières du quadrivium (à la différence de ce qui se passe à Oxford). Vous avez pourtant montré que cet enseignement des "mathématiques" (science des astres; mathématiques au sens propre) avait bien lieu mais dans un cadre privé, et qu'il s'agissait de leçons données chez les maîtres les jours de fêtes (vous avez même parlé un jour de "friandises de jours de fêtes "). Comment expliquer cette différence entre Oxford et Paris, d'une part, et les différences entre ces deux universités et les universités italiennes, de l'autre?

À Oxford, il y avait un enseignement scientifique qui dépassait ce qui est indiqué dans les statuts; et ces formes d'enseignement privé existaient également. Et à Bologne de même. Je pense que ce système d'enseignement privé et, par conséquent, de liberté du programme, était assez généralisé. Dans l'un de mes articles, j'ai cité ce statut de Heidelberg qui disait que pendant les jours de fête, il valait mieux que les étudiants disputent avec leur langue plutôt qu'ils ne se battent avec leurs dagues. La chose était assez généralisée et la publicité de cette affaire se trouvait relativement limitée du fait que cet enseignement du quadrivium débouchait fatalement sur l'enseignement de l'astrologie, ce qui était manié avec précaution ${ }^{22}$.

Comment expliquer l'officialisation de l'enseignement de l'astronomie/astrologie dans les universités italiennes et le fait que cela reste assez confidentiel à Oxford et à Paris?

À Paris, l'Université était dominée par la Faculté de théologie qui avait une sorte de fonction ecclésiale, reconnue par la papauté, d'être la gardienne de l'orthodoxie, le porte-parole de l'Église, ce qui créait des freins ou des conditions particulières. À Bologne, il y avait une domination des médecins, laquelle était beaucoup plus favorable à la reconnaissance de l'astrologie.

On pense à ce fameux statut de 1405 de Bologne, que vous connaissez bien et qui semble mirifique pour l'enseignement du quadrivium ${ }^{23}$. Pensez-vous que ce programme était purement théorique ou bien certains étudiants suivaient-ils vraiment ce cursus?

Il est difficile de répondre. La dernière fois que j'ai revu ces textes dans la conférence que j'ai faite à l'Institut Poincaré, j'ai quand même eu l'impression que la différence entre la réalité bolonaise et la réalité parisienne était moins grande qu'il ne semble à première vue ${ }^{24}$.

Dans un article déjà ancien, Tullio Gregory opposait une perception plus symbolique du monde dérivée de l'augustinisme et dominant au haut Moyen Âge et, à partir de la "Renaissance du XII siècle", une appréhension plus étiologique, plus scientifique en somme, de la nature qu'il attribuait tout d'abord à l'influence du Timée de Platon ${ }^{25}$. Vous avez étudié le symbolisme des nombres: doit-on établir un parallélisme entre le passage du nombre symbolique au nombre mathématique et ce changement de regard?

Mon intérêt pour la symbolique des nombres provenait essentiellement de la découverte d'un groupe de cisterciens du xiie siècle (Eudes de Morimond étudié depuis par Hanne Lange, Guillaume d'Obrives, Geoffroi d'Auxerre), qui m'avait orienté vers ce domaine d'étude ${ }^{26}$. Ce sont des personnages peu influencés par la "Renaissance" scientifique du xiie siècle et qui ne tiennent pas compte de l'apparition des chiffres arabes. C'est donc un peu décalé. Mon intérêt pour cette question était aussi aiguisé par un parallélisme possible avec le développement de l'intérêt pour les nombres chez les kabbalistes d'une période légèrement postérieure. Mais si l'on se place sur un plan général, on peut évidemment distinguer l'attitude vis-à-vis du nombre qui est lié à la dominance du platonisme augustinien dans les premiers siècles du Moyen Âge, et puis 
une attitude beaucoup plus détachée, résultant de l'influence de l'aristotélisme, qui diminue ce genre d'intérêt. Mais cela n'empêche pas que, quelles que soient les coupures chronologiques que l'on fait dans la pensée médiévale, il y a des archaïsmes, des survivances, qui sont souvent très vivaces.

Du nombre, passons à ce qu'Aristote définissait comme la " mesure du mouvement ", c'est-à-dire le temps. Vous avez écrit plusieurs contributions sur ce sujet. Dans votre exposé prononcé en 1993, à l'Académie des sciences morales et politiques, sur «le temps à l'aube de la science moderne", vous présentez avec prudence un parallélisme entre "la diffusion des horloges à poids" - les horloges mécaniques - et les nouvelles manières de concevoir le temps par les auteurs scolastiques à la fin $d u$ XIII et au début du XIV ${ }^{e}$ siècle $^{27}$. Nous aimerions que vous reveniez un peu sur ce sujet difficile mais très important.

Tout le monde n'est pas d'accord avec moi. L'apparition des horloges mécaniques pose un problème. Je fais allusion à la fameuse polémique entre Derek de Solla Price et des auteurs comme Jacques Le Goff, David Landes, etc., où il s'agit de savoir si les horloges mécaniques sont le résultat de la pression, d'une nécessité sociale ou bien si, au contraire, ces horloges sont apparues comme des espèces d'équatoires animés mécaniquement, dont le rôle était primitivement davantage lié à l'astrologie et à l'astronomie qu'aux conditionnements sociaux ${ }^{28}$. Mais il est évident que, quels que soient les enchaînements de causalité, apparaît de fait chez toute une série de scolastiques une conception du temps qui se détache de la définition aristotélicienne faisant $d u$ temps la mesure du mouvement, c'est-à-dire en fait la mesure du mouvement du ciel, pour devenir un écoulement autonome. Il y a un parallélisme entre le fait d'avoir un temps qui n'est plus lié directement au mouvement de la sphère céleste, qui prend son indépendance dans son écoulement vis-à-vis de celui-ci, d'une part, et, d'autre part, l'apparition d'un temps qui dépend d'une mécanique et qui par conséquent est lui-même indépendant du mouvement céleste. Les deux phénomènes ont un parallélisme qui est incontestable, mais le parallélisme lui-même n'entraîne pas obligatoirement l'intervention de relations causales. En plus, l'ouvrage de Gerhard Dohrn-van Rossum veut prouver que les premières horloges mécaniques étaient plus indépendantes de l'astronomie qu'on ne l'a dit ${ }^{29}$. Landes disait que les horloges qui étaient au sommet des clochers dès le début $d u$ xiii $<<>>$ siècle ne pouvaient pas être des horloges à eau parce que l'eau gelait l'hiver, et il tirait de là le fait que ces horloges étaient mécaniques. L'un des documents cruciaux sur ce point est un texte passionnant de 1271 ; il s'agit du commentaire de Robertus Anglicus sur le Traité de la sphère de Jean de Sacrobosco : ce texte est extraordinaire car c'est le premier témoignage sur une invention en train de se faire. Robertus Anglicus explique que l'on est sur le point d'inventer des horloges qui permettront de matérialiser le mouvement de la sphère céleste. Il dit que les magistri horologiorum sont sur le point d'y parvenir. L'interprétation de Landes était de dire que la mention de ces magistri horologiorum signifiait qu'il y avait déjà des corporations d'horlogers! Mais horologium peut très bien désigner, par exemple, un petit cadran cylindrique portatif, qui existe dès Hermann le Boiteux ; parfois le mot sert aussi à désigner les astrolabes...

Gerhard Dohrn-van Rossum remet en cause le travail de Jacques Le Goff sur le temps publié dans Pour un autre Moyen Âge, mais il ne résout pas le problème capital de savoir si les premières horloges mécaniques sont, comme il semble l'indiquer, des horloges publiques qui apparaissent dans les clochers en Italie à partir de 1300 environ, ou bien des instruments fabriqués par des 
astronomes en Angleterre dans des cathédrales dès les années 1280. Le problème a été déplacé par rapport au débat du temps de Price, mais au fond il n'est pas vraiment résolu.

J'ai en tout cas tendance à penser que l'invention de véritables horloges mécaniques à poids n'est pas antérieure à 1271 .

51 Abordons maintenant la question des marges de la science. Dans quelle mesure considérez-vous que l'histoire des sciences médiévales doit intégrer une réflexion et des recherches sur l'astrologie et l'alchimie qui ne font plus partie à l'heure actuelle de ce qu'il est convenu d'appeler « sciences »?

52 J'ai beaucoup milité pour cela. En particulier, lors du Congrès de Philosophie Médiévale qui a eu lieu à Bonn en 1977, j'ai organisé une réunion qui a eu un assez grand succès pour savoir si l'histoire de l'astrologie et de l'alchimie faisait partie de la philosophie médiévale. Un grand nombre de spécialistes se sont montrés intéressés ${ }^{30}$. Cela a contribué à un mouvement (que naturellement je n'ai pas créé) de meilleure acceptation de l'astrologie et de l'alchimie par les historiens des sciences et les historiens de la philosophie; parce que je me souviens d'un Congrès des Sociétés Savantes - je faisais à ce moment-là partie de la commission d'histoire des sciences du Comité des Travaux Historiques et Scientifiques - où, lorsqu'on avait proposé une communication sur l'histoire de l'astrologie dans la section "histoire des sciences", cela avait été une véritable levée de boucliers. Il y a donc là une évolution assez rapide, confortée par un certain nombre de très bonnes thèses universitaires. Il y a maintenant une tolérance plus grande ou une moins grande résistance aussi bien des historiens des sciences que des médiévistes à l'égard de ce genre d'études, qui semblaient alors non seulement marginales mais un petit peu fantaisistes.

Auparavant, le principal intérêt que l'on reconnaissait à l'alchimie et à l'astrologie résidait dans les résultats que l'on pouvait intégrer à la science stricto sensu, par exemple, dans le cas de l'alchimie les découvertes de chimie. Or désormais on peut affirmer que c'est notamment sur le plan intellectuel, conceptuel, que l'alchimie et l'astrologie sont importantes pour l'histoire des sciences.

Les cas de l'alchimie et de l'astrologie sont assez différents. Pour l'astrologie, je pense qu'il y a eu une date assez importante : la parution du livre de Richard Lemay sur Abû Ma'shar, qui a sensibilisé les philosophes au fait que les premiers apports de la philosophie naturelle d'Aristote se sont faits par le biais de textes astrologiques et que, par conséquent, si les textes d'astrologie étaient les véhicules de l'aristotélisme, il fallait les intégrer à l'histoire de cet ensemble de doctrines ${ }^{31}$. Moi-même, $\mathrm{j}$ 'ai beaucoup soutenu au CNRS, lorsque j'étais membre de la commission, les travaux de Richard Lemay.

55 Aujourd'hui, du reste, les progrès très grands qui sont faits dans les éditions de textes contribuent à une meilleure connaissance des doctrines. On peut penser pour l'astrologie aux travaux de Charles Burnett et d'autres, et pour l'alchimie aux travaux de Sylvain Matton, Didier Kahn et du groupe de Chrysopoeia qui font considérablement avancer ces domaines.

Pour l'alchimie, le fascicule de Robert Halleux sur la typologie des sources alchimiques a eu un rôle utile pour convaincre les historiens médiévistes qui ne s'intéressaient pas à l'alchimie que cela faisait partie des choses essentielles qu'il fallait appréhender ${ }^{32}$. En Allemagne aussi, les travaux de Joachim Telle ont été importants. 

pense à ce sujet, bien sûr les traducteurs du XII siècle et Tolède viennent à l'esprit, mais aussi Alphonse X el Sabio... le sage ou le savant? «savant» et «sage ». Dans le cas d'Alphonse X, comme il était fort peu sage et que c'était un politique absolument désastreux, alors l'interprétation a posteriori n'est pas douteuse... Il faudrait épiloguer sur les mots sapientia et sapiens qui veulent moins souvent dire «sagesse » et "sage » que ne le pensent la plupart des gens. Beaucoup plus souvent sapiens veut dire "savant». Il y a évidemment cette idée philosophique que le savoir rend sage, ce que nous savons tous par expérience n'être pas toujours confirmé...

D'Alphonse X passons aux tables alphonsines. Que pensez-vous des travaux récents de John North, José Chabás et Bernard Goldstein, qui relativisent la portée innovatrice de la version latine des tables dites alphonsines ${ }^{33}$ ? Et quels enseignements en tirez-vous sur la nature et le rythme des progrès en astronomie dans les derniers siècles du Moyen Âge? Estimez-vous, à l'instar d'Emmanuel Poulle ${ }^{34}$, qu'il y a eu une véritable révolution dans la science astronomique avec l'apparition des tables latines attribuées à Alphonse X en 1320 à Paris, ou voyez-vous cela comme une évolution plus hésitante et comme un triomphe moins absolu de l'astronomie alphonsine à partir de 1320 ?

60 La révolution des tables alphonsines est davantage une révolution dans l'ordre de la présentation que dans l'ordre du contenu fondamental. C'est une présentation beaucoup plus facile, mais avec les tables de Tolède, à condition de bien s'en servir, on arrivait à des résultats qui n'étaient pas tellement différents (surtout qu'il y a une incertitude sur le mouvement d'accès et de recès de la huitième sphère, celle des étoiles fixes). Si l'on considère l'évolution de l'astronomie à la fin du Moyen Âge, les progrès dans la précision des calculs ne sont pas tellement manifestes. Il s'agit là avant tout d'une affaire de présentation.

61 En ce qui concerne les tables alphonsines, je ne veux pas polémiquer contre Emmanuel Poulle, qui est l'un de mes meilleurs amis, mais je ne suis pas totalement convaincu par sa théorie pour une raison que, en dehors de toute polémique, je limite à quelque chose de ponctuel. Comme vous le savez, j'ai découvert à l'Escorial un manuscrit d'un intérêt considérable dans la mesure où il s'agit d'un manuscrit universitaire normal par son contenu (le corpus de Sacrobosco, le quadrant, le traité de l'astrolabe attribué à Messahalla, Thâbit ibn Qurra, etc.) mais très luxueux, avec de grandes marges blanches ${ }^{35}$. Or c'est un manuscrit dont j'ai découvert qu'il avait appartenu à Jean de Murs, et comme il y avait beaucoup de parchemin blanc dans ce codex astronomique, il l'a utilisé comme une espèce d'agenda pour noter ses impressions au jour le jour et, en particulier, pour noter ses observations en calculant à l'avance l'heure des éclipses et en s'interrogeant pour savoir comment s'expliquait la petite différence qu'il pouvait trouver entre l'heure prévue et l'heure réelle. Et avec un esprit critique assez remarquable, qui est de l'ordre de la confidence puisque ce n'était pas fait pour être publié, il s'interroge sur la question de savoir si les discordances entre la prévision et l'observation s'expliquent par l'erreur des tables ou si cela s'explique par l'erreur de l'observation ou par la manière dont on utilise les tables. Ces calculs ne mettent donc pas du tout en cause le rôle plus ou moins clandestin des tables alphonsines dans la réforme de l'astronomie puisque, dès le début, Jean de Murs utilise des tables qu'il donne comme Tabule regis Alphonsi, ce qui serait un peu curieux si c'était lui qui les 
avait fabriquées. Et là où il y a un problème qui met tout de même Poulle en difficulté, c'est que ce calcul d'après les Tabule regis Alphonsi part de tables de conjonctions moyennes du soleil et de la lune, alors que, dans l'édition des tables alphonsines qu'a donnée Poulle, il n'y a pas de tables des conjonctions moyennes du soleil et de la lune. Cela me laisse donc penser que Jean de Murs considérait lui-même comme «tables du roi Alphonse » des tables qui ne sont pas exactement celles qu'a publiées Poulle et qui comportaient cette partie. Poulle a fait l'édition de ces tables en se fondant sur l'édition princeps, publiée à Venise en 1483, mais de très grandes variantes existent entre les nombreux manuscrits, et dans la thèse sur Jean de Lignères de Marie-Madeleine Saby, il $\mathrm{y}$ a des traces de ces tables de conjonctions moyennes des tables alphonsines ${ }^{36}$. Si bien qu'en réalité, on ne connaît pas aussi bien ces tables que l'on croit; et je pense qu'il a dû venir à Paris des espèces de résidus, de moignons, de fragments des tables alphonsines castillanes sur la base desquelles Jean de Murs et Jean de Lignères ont pu travailler pour en faire une édition. Jean de Murs parle avec une espèce de respect de ces Tabule regis Alphonsi, et, quel que soit son sens de l'humour, bien connu par ailleurs - il y a en effet un aspect un peu chansonnier chez Jean de Murs : il avait écrit une lettre à l'astrolabe, laquelle est une espèce de canular, et Poulle tire argument de cela -, je pense que Jean de Murs a utilisé des tables de conjonctions moyennes du soleil et de la lune qu'il considérait de bonne foi comme étant réellement du roi Alphonse. Il faudrait faire un ratissage systématique des manuscrits et publier, si possible, une édition critique de ce qu'est réellement la version originelle des tables alphonsines latines.

Comment expliquer la place et le rôle des savants juifs dans la science espagnole au Moyen Âge et notamment dans les domaines médical et médico-astrologique?

Le problème n'est pas seulement celui des savants juifs, mais celui des savants ibériques tout court. Lorsque j'ai démarré ma quête des manuscrits scientifiques conservés en Espagne, je considérais, avec les idées que j'avais encore de mon passage à l'École des chartes, que de ces manuscrits allait sortir une histoire de la science médiévale en Espagne. Or je me suis heurté au fait que, en raison de la grande richesse de l'Espagne au $\mathrm{xvi}^{\mathrm{e}}$ siècle, une majorité de manuscrits acquis à cette époque provenait d'autres régions d'Europe. Je cite assez souvent le mot d'Emilio García Gomez, selon lequel pour les Espagnols, la science arabe a agi à la fois comme un aliment et comme un révulsif. Il $\mathrm{y}$ a là un problème de mentalité assez intéressant où les savants espagnols - cela vaut aussi pour les savants européens mais, du fait de l'esprit de Reconquista, c'est plus important en Espagne - sont absolument fascinés par la supériorité de la science arabe et sont en même temps marqués par une hostilité et une volonté de Reconquête vis-àvis du monde arabe. Les savants juifs ont évidemment joué un grand rôle à la cour d'Alphonse X, mais le cas de cette cour est assez particulier.

À la cour de Pierre IV le Cérémonieux il y a une intervention assez importante des savants juifs dans les tables astronomiques dites de Pierre d'Aragon; vous avez cité à l'occasion de la publication du livre de Maxime Préaud le magnifique dialogue entre l'astrologue Abraham ibn Zarsal et Pierre le Cruel, en 1369 ; il y a aussi l'exemple fameux de l'opération de la cataracte de Jean II d'Aragon en 1468, où un médecin et astrologue juif est invité à opérer ${ }^{37}$. Tout cela n'est-il pas l'indice d'un rôle important joué par les savants juifs en Espagne jusqu'en 1492 ?

65 Ces savants juifs en Espagne continuent assez largement à savoir l'arabe, ce qui est une supériorité. Et il est vrai aussi que dans le domaine de l'astrologie, ils ont une place particulière. Mais dans le domaine médical, il y a quand même une médecine latine 
assez importante avec de grands médecins, en particulier en Catalogne, comme l'ont montré notamment les dépouillements systématiques qu'a faits Michael McVaugh dans les archives de la Couronne d'Aragon ${ }^{38}$. Ce rôle des savants juifs a été conforté par la relative faiblesse des structures universitaires en Espagne. Et cela rejoint la question que vous posiez sur Guadalupe : l'originalité de Guadalupe s'explique en partie par cette faiblesse des structures universitaires. Il est évident qu'un couvent situé à quelques kilomètres de Montpellier n'aurait pas eu les mêmes avantages et les mêmes inconvénients.

À la différence des manuscrits des bibliothèques espagnoles auxquels vous vous référiez tout à l'heure, les manuscrits de Salamanque que vous avez beaucoup étudiés sont d'origine locale ${ }^{39}$...

Le gros avantage de ces manuscrits, c'est qu'ils proviennent de deux Colegios mayores, en particulier celui de Saint-Barthélemy; et il se trouve que l'un des utilisateurs de ces manuscrits est quelqu'un qui, pour avoir pris le parti de la Beltraneja, s'était réfugié au Portugal. Vous savez qu'il y a eu un problème délicat de succession: Henri IV étant considéré comme impuissant, sa fille était considérée comme illégitime, si bien que si l'on ne donnait pas la succession à sa fille, on la donnait à sa tante, Isabelle la Catholique, d'où une guerre civile assez violente qui opposa Isabelle la Catholique à la Beltraneja, laquelle était soutenue par les Portugais. Or l'un des utilisateurs de ces manuscrits au collège Saint-Barthélemy, qui était un mathématicien et un astrologue, avait pronostiqué la victoire de la Beltraneja et des Portugais contre Isabelle la Catholique. Évidemment c'était une prévision astrologique assez hasardeuse et malheureuse... et il a été obligé de se réfugier au Portugal, où il s'est imposé par sa notoriété scientifique. Il a fait partie de la commission (qui est un peu comme une commission du CNRS) qui a été chargée d'examiner le projet de Christophe Colomb. Il est donc intéressant de savoir quelle était la culture scientifique de l'un des principaux commissaires qui a refusé le projet de Colomb. Effectivement, ils avaient raison : ils ne se sont pas du tout ridiculisés en le refusant, parce que si l'Amérique n'avait pas existé et que Christophe Colomb avait dû traverser à la fois l'Atlantique et le Pacifique, il n'aurait pas acquis la célébrité dont il bénéficie indûment aujourd'hui... Cela donne donc une idée des connaissances auxquelles pouvaient avoir accès les responsables scientifiques. Cette Junta dos matematicos était vraiment une commission scientifique pour examiner le projet. Et il y a là une connexion entre théorie et pratique qui est particulièrement intéressante.

Quelle est la réalité du secret qui était devenu un mythe sous la plume d'une certaine historiographie portugaise?

Le secret n'est pas uniquement un mythe, mais l'on a profité de cette histoire du secret pour dire n'importe quoi. Le problème est donc plutôt méthodologique. Il est sûr qu'il y a un ou deux cas où l'on a vu Jean II de Portugal reprocher à des gens d'avoir eu la langue trop bien pendue sur les données scientifiques. Une fois, quelqu'un a même dit que, au fond, les caravelles ne servaient à rien sinon à s'échouer sur le rivage et que l'on pouvait faire la même chose avec d'autres bateaux. Après que cela eut été dit devant des oreilles éventuellement indiscrètes, Jean II s'est trouvé seul avec l'auteur de cette indiscrétion, et il l'a fortement sermonné !

Quel est le rôle de la science médiévale, et en particulier de l'astronomie, dans les grandes découvertes maritimes? Il semble tardif, postérieur en tout cas aux premières grandes découvertes portugaises. 
71 Il y a toutes sortes d'autres éléments que la navigation astronomique. Avant l'époque où l'on a été capable de calculer, en mer, la latitude correcte avec un instrument astronomique, il existait une navigation pré-astronomique où l'on était capable, non de déterminer des chiffres absolus de latitude, mais de prendre des différences de latitudes. Et puis il y a aussi ce problème : quand on a un vent défavorable et que l'on s'éloigne de la ligne de rhumb indiquée par une carte et que l'on dévie de sa trajectoire, il faut être capable de calculer comment on va revenir sur sa trajectoire primitive, grâce à une trigonométrie rudimentaire. À partir $\mathrm{du}$ xiii ${ }^{\mathrm{e}}<\mathrm{P}>$ siècle, un certain nombre d'utilisations scientifiques des mathématiques et de l'astronomie se sont développées. Et cela a été l'utilité du dialogue que nous avons eu avec les Portugais : il ne fallait pas dire que les Catalans naviguaient $a u x \mathrm{xiv}^{\mathrm{e}}<\mathrm{P}>$ siècle comme on navigue au $\mathrm{Xvi}^{\mathrm{e}}$ siècle ; il a fallu retrouver les divers biais qui ont permis de réaliser des connexions entre les connaissances scientifiques et certains procédés empiriques mis en pratique ; et c'est une chose très complexe.

Comment définiriez-vous la science au Moyen Âge?

un des derniers congrès de la Société Internationale pour l'Étude de la Philosophie Médiévale qui a eu lieu à Erfurt en 1997, j'avais organisé une réunion autour de la question: "L'histoire des sciences est-elle, pour le Moyen Âge, porteuse d'anachronismes psychologiques? $»^{40}$. C'est un sujet difficile. On peut dire d'un côté que la notion de science est porteuse d'anachronismes dans la mesure où il s'agit de philosophie naturelle plus que de science; mais, d'un autre côté, cela n'est pas vrai pour tout: le statut épistémologique des mathématiques, par exemple, n'est pas tellement différent au Moyen Âge de ce qu'il peut être pour une période postérieure.

Quand on est confronté à la question de la science médiévale - et Danielle Jacquart a souvent abordé ce problème dans le séminaire qui a pris le relais du vôtre à la IV section de l'École Pratique des Hautes Études -, on rencontre la difficulté liée à l'écart entre l'acception médiévale du terme scientia et l'acception moderne du mot "science ».

Cette question rejoint évidemment celle qui concernait l'extension de la Révolution scientifique. La plupart d'entre nous ont opté pour cette solution facile, mais qui n'est pas mauvaise, de parler de savoir scientifique plutôt que de science.

\section{NOTES}

1. Voir notamment G. Beaujouan, « Recherches sur l'histoire de l'arithmétique au Moyen Âge ", École nationale des chartes, positions des thèses, 1947, p. 17-22 ; Id., « L'enseignement de l'arithmétique élémentaire à l'Université de Paris aux xiii ${ }^{\mathrm{e}}$ et xiv ${ }^{\mathrm{e}}$ siècles ", dans Homenaje a Millàs Vallicrosa, vol. 1, Barcelone, 1954, p. 93-124, art. réimpr. dans Id., Par raison de nombres. L'art du calcul et les savoirs scientifiques médiévaux, Aldershot, 1991, texte $n^{\circ}$ XI ; Id., " The Place of Nicolas Chuquet in a Typology of Fifteenth-Century French Arithmetics », dans C. Hay (éd.), Mathematics from Manuscript to Print, Oxford, 1988, p. 73-88, art. réimpr. dans Id., Par raison de nombres, op. cit., texte 
$\mathrm{n}^{\circ}$ III ; Id., « Nombre », dans J. Le Goff, J.-C. Schmitt (dir.), Dictionnaire raisonné de l'Occident médiéval, Paris, 1999, p. 834-844.

2. G. Beaujouan, «Incompréhension entre historiens des sciences et historiens (le cas du Moyen Âge) » dans L'histoire des sciences et des techniques doit-elle intéresser les historiens? [colloque organisé par la Société française d'histoire des sciences], Paris, 1982, p. 16-20.

3. C.-V. Langlois, La connaissance de la nature et du monde au Moyen Âge, d'après quelques écrits français à l'usage des laïcs, Paris, 1928, réimpr. Genève, 1970.

4. C. Thomasset, Une vision du monde à la fin $d u$ XIII siècle : commentaire du dialogue de Placides et Timeo, Genève, 1982.

5. Voir notamment P. Duhem, Le système du monde. Histoire des doctrines cosmologiques de Platon à Copernic, 10 vol., Paris, 1913-1959 ; A. Koyré, Du monde clos à l'univers infini, Paris, 1962 (nouv. éd. Paris, 1988).

6. A. Maier, Die Vorlaüfer Galileis im 14. Jahrhundert, Rome, 1949.

7. G. Beaujouan, « Alexandre Koyré, l'évêque Tempier et les censures de 1277 », History and Technology, vol. 4 (1987), p. 425-429, art. réimpr. dans Id., Par raison de nombres, op. cit., texte $\mathrm{n}^{\mathrm{O}} \mathrm{VI}$.

8. Voir notamment E. GRANT, «The Condemnation of 1277 , God's Absolute Power and Physical Thought in the Late Middle Ages », Viator, 10 (1979), p. 211-244 ; ID., Planets, Stars, and Orbs. The Medieval Cosmos, 1200-1687, Cambridge, 1994.

9. H. Hugonnard-Roche, L'œuvre astronomique de Thémon Juif, maître parisien du XIV siècle, Genève/Paris, 1973.

10. W. A. WAllace, Domingo de Soto and the Early Galileo, Aldershot, 2004 ; A. C. CROMBIE,

A. CARUGO, "The Jesuits and Galileo's Ideas of Science and of Nature ", Annali dell'Istituto e Museo di Storia della Scienza di Firenze, viii-2 (1983), p. 3-68, réimpr. dans ID., Science, Art and Nature in Medieval and Modern Thought, Londres, 1996, p. 165-229.

11. Voir en particulier G. Beaujouan, « Réflexions sur les rapports entre théorie et pratique au Moyen Âge ", dans J. E. Murdoch et E. D. Sylla (éd.), The Cultural Context of Medieval Learning, Dordrecht, 1975, p. 437-484.

12. G. Beaujouan et $\mathrm{E}$. Poulle, "Les origines de la navigation astronomique aux xiv et $\mathrm{Xv}^{\mathrm{e}}$ siècles ", dans Le navire et l'économie maritime : travaux du Colloque d'histoire maritime, le 17 mai 1956, présentés par M. Mollat, Paris, 1957, p. 102-117.

13. Voir notamment $\mathrm{G}$. Beaujouan, "Science livresque et art nautique au $\mathrm{Xv}^{\mathrm{e}}$ siècle ", dans Les aspects internationaux de la découverte océanique aux $\mathrm{XV}^{e}$ et XVI siècles. Actes du $V^{e}$ colloque international d'histoire maritime, Paris, 1966, p. 61-85, réimpr. dans Id., Science médiévale d'Espagne et d'alentour, Aldershot, 1992, texte $\mathrm{n}^{\circ}$ IX.

14. Id., L'interdépendance entre la science scolastique et les techniques utilitaires (XII ${ }^{e}, X^{e} I^{e}$ et XIV siècles), Paris, 1957.

15. B. Gille, Les ingénieurs de la Renaissance, $2^{\mathrm{e}}$ éd., Paris, 1978.

16. G. Beaujouan, «Teoria e pratica nel Medioevo », dans Storia della scienza, vol. IV, Rome, 2003, p. 561-568.

17. Id., "Calcul d'expert, en 1391, sur le chantier du Dôme de Milan », Le Moyen Âge, 79 (1963), p. 555-563, réimpr. dans Id., Par raison de nombres, op. cit., texte $n^{\circ}$ XV.

18. Id., " La bibliothèque et l'école médicale du monastère de Guadalupe à l'aube de la Renaissance ", dans G. Beaujouan, Y. Poulle-Drieux et J.-M. Dureau-Lapeyssonnie, Médecine humaine et vétérinaire à la fin du Moyen Âge, Genève, 1966, p. 367-468. 
19. G. Beaujouan, «La prise de conscience de l'aptitude à innover (le tournant du milieu du xiiie siècle) », dans B. Ribémont (éd.), Le Moyen Âge et la science, Paris, 1991, p. 5-14 ; Id., «L'émergence médiévale de l'idée de progrès », Bulletin de philosophie médiévale, 30 (1988), p. 20-36.

20. Ibid.

21. Id., « Le quadrivium à la Faculté des arts », dans $\mathrm{O}$. Weijers et L. Holtz (éd.), L'enseignement des disciplines à la Faculté des arts (Paris et Oxford, XIII ${ }^{e}$-XVe siècles), Paris, 1997, p. 185-194.

22. Id., "Motives and Opportunities for Science in the Medieval Universities ", dans A. C. Crombie (éd.), Scientific Change, Londres, 1963, p. 219-236.

23. Sur ce statut, voir en dernier lieu G. Federici Vescovini, « I programmi degli insegnamenti del collegio di medicina, filosofia e astrologia, dello statuto dell'Università di Bologna del 1405 ", dans Roma, magistra mundi. Itineraria culturce medievalis. Mélanges offerts au Père L. E. Boyle, Louvain-la-Neuve, 1998, vol. I, p. 193-223. 24. Conférence inédite, 1999.

25. T. Gregory, "La nouvelle idée de nature et de savoir scientifique au xii e siècle ", dans J. E. Murdoch et E. D. Sylla (éd.), The Cultural Context of Medieval Learning, op. cit., p. 193-218.

26. G. Beaujouan, «Le symbolisme des nombres à l'époque romane », Cahiers de civilisation médiévale, 15 (1961), p. 159-169, réimpr. dans Id., Par raison de nombres, op. cit., texte $\mathrm{n}^{\circ} \mathrm{X}$.

27. Id., « Le temps à l'aube de la science moderne », Revue des sciences morales et politiques, 1993, p. 67-75.

28. D. J. de Solla Price, « Clockwork before the Clock », Horological Journal, 1955, p. 810-814, 1956, p. 31-35 ; J. Le goff, « Au Moyen Âge : temps de l'Église et temps du marchand ", art. de 1960 réimpr. dans Id., Pour un autre Moyen Âge : temps, travail et culture en Occident : 18 essais, Paris, 1977, p. 46-79 ; D. S. Landes, Revolution in Time : Clocks and the Making of the Modern World, Cambridge (Mass.), 1983, trad. fr. : L'heure qu'il est : les horloges, la mesure du temps et la formation du monde moderne, Paris, 1987.

29. G. Dohrn-van Rossum, L'histoire de l'heure. L'horlogerie et l'organisation moderne du temps, Paris, 1997, trad. de Die Geschichte der Stunde. Uhren und moderne Zeitordnung, Munich, 1992.

30. G. Beaujouan, Rapport sur le thème : « En quoi les recherches sur l'astrologie et l'alchimie peuvent-elles améliorer notre compréhension de la philosophie médiévale ? ", dans A. ZimmERMAnN (éd.), Miscellanea mediaevalia... des Thomas-Instituts Univ. Köln, 13/1, Sprache und Erkenntnis im Mittelalter, Berlin-New York, 1981, p. 114-127. 31. R. LEMAY, Abu Ma'shar and Latin Aristotelianism in the 12th Century. The Recovery of Aristotle's Natural Philosophy through Arabic Astrology, Beyrouth, 1962.

32. R. Halleux, Les textes alchimiques, Turnhout, 1979 (Typologie des sources du Moyen Âge occidental, fasc. 32).

33. Voir notamment J. NORTH, « Just whose were the Alfonsine Tables? ", dans From Baghdad to Barcelona. Studies in the Islamic Exact Sciences in Honour of Prof. Juan Vernet, Barcelone, 1996, vol. I, p. 453-475, et en dernier lieu J. Chabás et B. Goldstein, The Alfonsine Tables of Toledo, Dordrecht/Boston/Londres, 2003.

34. E. Poulle, Les tables alphonsines avec les canons de Jean de Saxe, édition, traduction et commentaire, Paris, 1984 ; Id., " Les tables alphonsines et Alphonse X de Castille », Académie des Inscriptions et Belles Lettres. Comptes rendus des séances, 1987, p. 82-102. Les tables astronomiques latines dites alphonsines ont supplanté, à partir de 1320, les 
tables de Tolède qui avaient été adaptées à différents lieux de la chrétienté. Emmanuel Poulle considère que ces tables ne proviennent pas, en réalité, de l'entourage du roi de Castille Alphonse X (1252-1284), mais qu'elles constituent une création originale des astronomes parisiens Jean de Murs et Jean de Lignères. North, Chabás et Goldstein défendent la paternité alphonsine et pensent que la version latine dépend d'un original castillan.

35. G. Beaujouan, « Observations et calculs astronomiques de Jean de Murs (1321-1344) ", dans 14th International Congress of the History of Science. Proceedings $\mathrm{n}^{\circ} 2$, Tokyo, 1975, p. 27-30 ; article réimpr. dans Id., Par raison de nombres, op. cit., texte $\mathrm{n}^{\circ}$ VII. 36. M.-M. Saby-Rousset, Les canons de Jean de Lignères sur les tables astronomiques de 1321. Édition critique, traduction et étude, thèse d'École des chartes, Paris, dactyl., 1987, sous presse. Voir en attendant M.-M. Saby-Rousset, « Les canons de Jean de Lignères... », École nationale des chartes, positions des thèses, 1987, p. 183-190.

37. Pour une synthèse sur ce sujet, voir G. Beaujouan, La science en Espagne aux XIV et $X V^{e}$ siècles, Paris, 1967 ; Id., «L'astronomie dans la péninsule ibérique à la fin du Moyen Âge ", Agrupamento de estudos de cartografia antiga, XXIV (1969), p. 3-22, réimpr. dans Id., Science médiévale, op. cit., texte $\mathrm{n}^{\circ} \mathrm{X}$.

38. M. McVAugh, Medicine before the Plague: Practitioners and their Patients in the Crown of Aragon, 1285-1345, Cambridge, 1993.

39. G. Beaujouan, Manuscrits scientifiques médiévaux de l'Université de Salamanque et de ses " colegios mayores ", Bordeaux, 1962 (Bibliothèque des Hautes Études Hispaniques, vol. 32).

40. Le compte rendu de cette réunion a été publié dans le Bulletin de philosophie médiévale, 39 (1997), p. 23-30. 